\title{
A Review of the Textbook Osnovy dinamicheskoi biokhimii (Fundamentals of Dynamic Biochemistry) by V. K. Plakunov and Yu. A. Nikolaev (Moscow: Logos, 2010)
}

DOI: $10.1134 / \mathrm{S} 0026261711020068$

Since no textbook presently exists aimed at graduate students and PhD students without basic biochemical training, this publication is certainly a timely event.

The authors are established scientists working on the biochemical mechanisms of metabolic regulation and possess extensive teaching experience. They have been lecturing for years in the leading Russian universities, such as Moscow State University and Mendeleev University of Chemical Technology of Russia.

This textbook, the result of creative interpretation of the authors' scientific and educational experience, is in fact a treatise on the current state of the major biochemical problems. Listing the titles of the main sections of the book will suffice to show its scope:

Chapter 1. Static Biochemistry of the Cell.

Chapter 2. Fundamentals of Enzymology.

Chapter 3. Organization of Metabolic Processes.

Chapter 4. Regulation of Metabolic Processes.

Chapter 5. Mechanisms of Global Regulation of Metabolic Processes.

Chapter 6. The Role of Biochemistry for Biotechnology.

The textbook is well written in clear language. The illustrations and examples, including those related to practical aspects of the regulation of enzyme biosynthesis and their technological application, are useful for understanding the material.

The description of the methods for enzyme purification and assessment of enzyme specificity will be useful both for students and for people who work in the biotechnological industry.

The section on enzyme kinetics and the mechanisms of inhibition and stimulation of enzymatic activity is highly useful, especially for students and young researchers.
The authors provide a detailed, up-to-date description of the processes of membrane transport, which is one of the fields of their own research. In addition to traditional models of nutrient inflow into the cell, this section (Chapter 3) deals with the mechanisms of metabolic regulation based on reverse transport of the substrates and products from the cell. The authors consider these mechanisms as a specific level of metabolic regulation, terming it membrane regulation.

This is the first textbook to consider the role of global mechanisms of regulation of microbial metabolism, in particular, the mechanisms of "oxygen regulation," and the role of microbial biofilms in environmental and biotechnological processes.

The textbook contains a great amount of information on biochemistry and molecular biology.

Although less detailed than the many volumes of translated biochemical monographs, it will be useful as a fundamental textbook on biochemistry for researchers and student studying biology, ecology and nature management, or chemical technology and biotechnology (the specializations Biology, Physiology, Microbiology, Biotechnology, and Ecobiotechnology). As an aid in biochemical self-education, the book will be of interest to specialists, researchers, and graduate and Ph.D. students.

Unfortunately, the low number of copies limits its availability to specialists and students. It is practically not being distributed through bookstores.

In subsequent editions, the authors should expand the list of recommended literature, including the latest textbooks by Varfolomeev et al. and others.

The textbook may be purchased directly from the Logos publishing house (phone/fax: 7-(095) 3695819, 3695668, 3697727, by e-mail: universitas@mail.ru) or via online bookshops.

N.B. Gradova 